\title{
Key histone chaperones have distinct roles in replisome progression and genomic stability
}

Ioannis Tsirkas ${ }^{1}$, Daniel Dovrat ${ }^{1}$, Yang Lei ${ }^{2}$, Angeliki Kalyva ${ }^{1}$, Diana Lotysh ${ }^{1}$, Qing Li $^{2}$ and Amir Aharoni $^{1 *}$

${ }^{1}$ Department of Life Sciences and the National Institute for Biotechnology in the Negev, Ben-Gurion University of the Negev, Be'er Sheva 84105, Israel. ${ }^{2}$ State Key Laboratory of Protein and Plant Gene Research, School of Life Sciences and Peking-Tsinghua Center for Life Sciences, Peking University, Beijing 100871, China

*Correspondence should be addressed to A.A. (aaharoni@bgu.ac.il) 


\begin{abstract}
Replication-coupled (RC) nucleosome assembly is an essential process in eukaryotic cells in order to maintain chromatin structure during DNA replication. The deposition of newly synthesized H3/H4 histones during DNA replication is facilitated by specialized histone chaperones. Although the contribution of these histone chaperones to genomic stability has been thoroughly investigated, their effect on replisome progression is much less understood. By exploiting a time-lapse microscopy system for monitoring DNA replication in individual live cells, we examined how mutations in key histone chaperones including CAC1, RTT106, RTT109 and $A S F 1$, affect replication fork progression. Our experiments revealed that mutations in CAC1 or RTT106 that directly deposit histones on the DNA, slowdown replication fork progression. In contrast, analysis of cells mutated in the intermediary ASF1 or RTT109 histone chaperones revealed that replisome progression is not affected. We found that mutations in histone chaperones including ASF1 and RTT109 lead to extended G2/M duration, elevated number of RPA foci and in some cases, increased spontaneous mutation rate. Our research suggests that histone chaperones have distinct roles in enabling high replisome progression and maintaining genome stability during cell cycle progression.
\end{abstract}

\title{
Author Summary
}

Histone chaperones (HC) play key roles in maintaining the chromatin structure during DNA replication in eukaryotic cells. Despite extensive studies on HCs, little is known regarding their importance for replication fork progression during S-phase. Here, we utilized a live-cell imaging approach to measure the progression rates of single replication forks in individual yeast cells mutated in key histone chaperones. Using this approach, we show that mutations in $C A C 1$ or RTT106 HCs that directly deposit histones on the DNA lead to slowdown of replication fork progression. In contrast, mutations in $A S F 1$ or RTT109 $\mathrm{HCs}$ that transfers $\mathrm{H} 3 / \mathrm{H} 4$ to $C A C 1$ or RTT106, do not affect replisome progression but lead to post replication defects. Our results reveal distinct functions of HCs in replication fork progression and maintaining genome stability. 


\section{Introduction}

DNA replication in eukaryotic cells is a complex process that requires the accurate copying of the DNA itself and the formation of a precise chromatin structure $[1,2]$. The basic unit of chromatin is the nucleosome, composed of $\sim 146$ base pairs of DNA wrapped around an octamer of histones. A nucleosome is composed of a core of $(\mathrm{H} 3-\mathrm{H} 4)_{2}$ tetramer and two flanking $\mathrm{H} 2 \mathrm{~A}-\mathrm{H} 2 \mathrm{~B}$ dimmers $[3,4]$. During DNA replication, nucleosomes must be disassembled to allow replication fork progression and subsequently must be reassembled to establish the accurate chromatin state. Histone chaperones are essential for the process of DNA replication-coupled (RC) nucleosome deposition by facilitating correct histone assembly, post-translational modifications and localization during DNA replication [5-8].

Newly synthesized histones are assembled into nucleosomes by several histone chaperones including Asf1, Rtt109, Rtt106, Caf1 and the FACT complex that act in a sequential and coordinated manner to facilitate nucleosome deposition during DNA replication [5,9-11]. In budding yeast, Asf1 binds newly synthesized H3-H4 histones and promotes H3K56 acetylation by Rtt109 [12]. Acetylation of H3K56 enhances H3-H4 binding to Caf1 and Rtt106 that deposit (H3/H4) $)_{2}$ tetramers directly onto the DNA [13-15]. Previous studies have shown that single- or double-mutations of histone chaperone genes including Caf1 subunits, RTT106, ASF1 or RTT109 can lead to severe replication stress, checkpoint activation, increased recombination and sensitivity to DNA damaging agents $[14,16,17]$. However, the effect of histone chaperone mutations on the rate of replisome progression is much less understood. While studies in human cells have shown that mutations in genes essential for the synthesis of histones significantly slowdown replisome progression [18], studies in yeast show that mutations in histone chaperones can have little effect on S-phase progression $[14,17,19-21]$.

One of the most important and extensively studied histone chaperone complex for RC nucleosome deposition both in vitro and in cells is the Cafl complex [13]. Caf1 is composed of three subunits, Cac1, Cac2 and Cac3, which are highly conserved from yeast to human [22]. Cac1 is the largest subunit of the complex and was shown to interact with Proliferating Cell Nuclear Antigen (PCNA) through a canonical PCNA Interacting Protein (PIP) motif [23]. Since PCNA is an essential subunit of the replisome, Cac1-PCNA interaction can couple between nucleosome deposition and DNA synthesis [24]. In addition, Cacl was shown to contain a Winged Helix Domain (WHD) motif allowing its direct binding to DNA [25]. While several biochemical studies provide important insights into the function of Caf1 subunits and the PIP and WHD domains of Cac1 [26,27], much 
less is known regarding their importance for replisome progression during S-phase and for nucleosome deposition on newly replicated DNA.

In this research, we have investigated the effect of deletion and/or mutations of major H3/H4 histone chaperones on DNA replication rate and G2/M duration. We have utilized our recently described live-cell microscopy approach [28,29] for the direct measurement of replisome progression and $\mathrm{G} 2 / \mathrm{M}$ duration in individual live yeast cells (see below for details). We found that deletion of $C A C 1$ or RTT106, which directly deposit nucleosomes on the DNA, lead to slowdown of replication fork progression. Further analysis of point mutations in Cac1 showed a separation of function in Cac1 WHD and PIP domains affecting replisome progression and G2/M duration, respectively. In addition, we found that deletion of $A S F 1$ or RTT109 histone chaperones that transfer $\mathrm{H} 3 / \mathrm{H} 4$ histones to $C A C 1$ and RTT106 did not lead to slowdown of replication fork progression but led to severe post-replication defects. Cell cycle analysis of the histone chaperone mutant cells, indicated a significant elongation of G2/M duration, ssDNA accumulation and, in some cases, elevated spontaneous mutation rates. These results demonstrate that histone chaperones exhibit distinct roles in facilitating high replisome progression rate and maintaining genome stability enabling efficient cell cycle progression.

\section{Results}

The experimental approach for measuring replication fork progression and G2/M duration in histone chaperone mutant strains

In order to examine the importance of Caf1, Rtt106, Asf1 or Rtt109 for replication fork progression during S-phase, we directly measured DNA replication rates in live WT and mutant cells [28,29]. Recently, we have described a live cell imaging approach for measuring the progression rates of single DNA replication forks in individual yeast cells [28]. This approach is based on the sitespecific integration of arrays of $l a c O$ and tetO bacterial operator sequences, bound by the respective GFP-lacI and tetR-tdTomato cognate repressors, allowing the labelling of specific chromosomal loci as two distinct fluorescent dots (Fig. 1). The fluorescent intensity of these dots increases during replication of the lacO and tetO arrays since more fluorescently labelled repressor proteins are recruited to the newly replicated arrays (Fig. 1). These arrays are utilized as reporters of replication fork progression by inserting one array adjacent to an early origin of replication and the other array downstream in the same replicon (Fig. 1). By monitoring replicating cells and quantifying the intensity of each dot over time using live-cell fluorescence microscopy, the replication time of each 
array region is determined. Since the distance between the arrays along the chromosome is known, fork progression rate can be derived. We recently demonstrated the applicability of this approach by examining replication at different loci, in the presence of different concentrations of $\mathrm{HU}$ and in different mutant strains [28]. More recently, we have utilized this approach to examine replication fork progression through G-quadruplex structures on the background of different pifl-mutant strains [29]. An additional advantage of this system is the ability to monitor yeast cell morphology and detect anaphase events at the single-cell level. Anaphase can be monitored as splitting of each fluorescent dot into two, one of which moves to the daughter cell. By calculating the time interval between replication of the second array $($ tet $O$ ) and anaphase, we can estimate the duration of $\mathrm{G} 2 / \mathrm{M}$ and examine how it is affected in different histone chaperone mutant cells (Fig. 1) [28].

\section{The importance of Caf1 complex subunits for replisome progression and G2/M duration:}

Previous studies have shown that Caf1 complex, composed of Cac1, Cac2 and Cac3, is important for $\mathrm{H} 3 / \mathrm{H} 4$ deposition directly onto the DNA[13,27]. To examine the importance of each subunit of Caf1 complex for replisome progression rate and $\mathrm{G} 2 / \mathrm{M}$ duration, we have deleted $C A C 1, C A C 2$ or $C A C 3$ subunits on the background of a strain containing the $\operatorname{lacO}$ and tet $O$ arrays located at the vicinity of ARS413. We then measured replication times and G2/M duration in individual yeast cells using live cell microscopy as described above (Fig. 2AB and Table S1). We found that cacldeletion leads to a significant elongation of replication times relative to the WT strain while cac2or cac3-deletion do not (Fig. 2C). Next, we analyzed the G2/M duration in the different strains and found that cacl-deletion cells exhibit significant increase in G2/M duration relative to WT cells (Fig. 2D). These results highlight the importance of Cac1 for replisome and G2/M progression and are in good agreement with a previous study showing that Cac1 is essential for the Caf1 protein complex assembly and H3/H4 binding [13,26].

Previous studies have revealed the contribution of the Cafl subunits for H3/H4 assembly and nucleosome deposition under DNA stress conditions [30-32]. To further investigate the effect of cac2- or cac3-deletion on DNA replication under these conditions, we incubated the Caf1 mutant strains with $20 \mathrm{mM} \mathrm{HU}$ and measured their replication times as described above. We found that all Caf1 component mutants exhibited longer replication times relative to WT (Fig. 2E and Table S2). These results are in agreement with previous studies [30-32] and deepen our understanding about the importance of Caf1 subunits on DNA replication under stress conditions.

\section{G2/M elongation due to cac1-deletion is linked to spindle checkpoint activation}


G2/M elongation may stem from the activation of two mitotic checkpoints due to genomic instability accumulated during DNA replication [33]. These two checkpoints are the DNA damage checkpoint and the spindle assembly checkpoint [34,35]. Previously, mutation in CAC1 was shown to be implicated in the activation of the spindle checkpoint [36]. Specifically, it was shown that the deletion of $M A D 2$, the master checkpoint protein of spindle assembly, suppressed the mitotic delay measured in cacl-and hirl-double deletion strain [36]. To examine whether the G2/M elongation observed in cac1-deletion cells (Fig. 2) was due to spindle checkpoint arrest, we deleted MAD2 in the background of cacl- deletion. We then measured replication times and G2/M duration in these mutant cells using the live-cell imaging system described above. In accordance with the previous study [36], we found that the elongation of G2/M duration due to $C A C 1$ deletion is suppressed in the double mutant cells and is similar to the G2/M duration values measured for WT cells (Fig. 3). To further support the importance of spindle checkpoint activation in cacl-deleted cells, we examined the growth of $\mathrm{cacl}$ - and mad2-double deleted cells using a spot assay. In agreement with the microscopy experiments, we found that the addition of $M A D 2$ deletion suppressed the growth defects observed in cacl-deleted cells (Fig. S1). These results, further supports the role of $C A C 1$ in ensuring mitotic spindle integrity prior to cell mitosis.

\section{Separation of function of the WHD and PIP regions in Cac1}

The elongation of replication times and G2/M duration of cacl-deleted cells can stem from the lack of Caf1 complex formation or from deficiencies in Cac1 specific interactions. Previously, Cac1 was shown to contain a WHD domain and a PIP region facilitating its direct interaction with the DNA and PCNA, respectively $[23,25]$. To examine the importance of these Cac1 regions for replisome progression and $\mathrm{G} 2 / \mathrm{M}$ duration, we have generated strains for replication measurements containing K564E/K568E and F233A/F234G mutations in Cac1 WHD domain and the PIP region, respectively. These mutations in the WHD and PIP domains of Cac1 were previously shown to abolish binding to DNA and PCNA, respectively [23,25]. Next, we measured replication time and G2/M duration in individual cells containing these mutations as described above. We found that mutating Cac1 WHD domain leads to increased replication times with no effect on the G2/M duration, relative to WT cells (Fig. 4A). In contrast, we found that mutating the PIP region of the protein does not affect replication times but leads to a significant elongation of G2/M duration (Fig. 4B). These results demonstrate the separation of function between the WHD and PIP regions of Cac1, with DNA binding contributing to proper replisome progression and PCNA binding preventing replication stress. 
Next, we examined the level of nucleosome deposition on newly synthesized DNA in the WHD and PIP Cac1 mutant strains at the vicinity of ARS305 and ARS605 using chromatin immunoprecipitation (ChIP) following release from HU arrest. In accordance with the effect of these mutations on replisome progression rate and G2/M duration, we found a significant decrease in the level of nucleosome deposition during DNA replication in these mutant strains relative to the WT (Fig. 4C-D and Fig. S2). To further examine the effect of HU replication stress on the two Cac1 mutants, we measured their replication times in presence of $20 \mathrm{mM} \mathrm{HU}$. We found that both mutants exhibit more than 9 minutes longer replication times compared to WT (Fig. S3). These results highlight the importance of both PIP and WHD of Cac1 for nucleosome deposition and replication fork progression at early S-phase under DNA stress conditions.

\section{Analysis of mutation in RTT106, RTT109 or ASF1 histone chaperones}

To examine how mutations in additional $\mathrm{H} 3 / \mathrm{H} 4$ histone chaperones affect replisome progression and G2/M duration, we have generated deletions in RTT106, RTT109 or ASF1 on the background of the $\operatorname{lac} O$ and tet $O$ containing strain as described above. In addition, to examine how double deletion in CAC1 and RTT106 affect these properties we have generated this strain on the same background. Rtt106 was previously shown to interact with $\mathrm{H} 3 / \mathrm{H} 4$ and the DNA to enable $(\mathrm{H} 3 / \mathrm{H} 4)_{2}$ tetramer deposition on newly synthesized DNA [37]. Cac1 and Rtt106 were shown to physically interact suggesting that these histone chaperones cooperate to facilitate high level of nucleosome deposition on newly synthesized DNA [38]. Asf1 and Rtt109 are additional key histone chaperone and histone acetylase, respectively, acting prior to Cac 1 and Rtt106 to facilitate H3K56 acetylation of newly synthesized histones [12,19]. Analysis of rtt106-deletion and the cacl-rtt106-double deletion cells using our assay showed significantly increased replication times, relative to WT cells. In contrast, rtt109- or asfl-deletion did not lead to a significant change in replication times (Fig. 5A). Analysis of the G2/M duration in cac1-rtt106-double deletion, rtt109- or asfl-deleted cells was significantly extended while was not affected in rtt106-deleted cells relative to WT cells (Fig. 5B). Elongation of G2/M duration, observed in cac1-rtt106-double deletion, rtt109- or asfl-deleted cells, is in agreement with several studies showing checkpoint activation and replication stress in these strains $[14,19-21]$.

\section{Increased G2/M duration in histone chaperone mutant cells is associated with ssDNA accumulation}

Replication stress often lead to the accumulation of single-stranded DNA near active replication and repair sites [39]. This accumulation can be visualized as foci of the ssDNA-binding protein 
Replication Protein A (RPA) [40-42]. To examine whether long G2/M duration in histone chaperone mutant cells is associated with increased level of ssDNA accumulation, we monitored the levels of RPA foci in these mutant cells. For monitoring RPA foci in live cells, we used Rfa1, expressed under its native promoter, tagged with GFP at its N-terminus [43] and used fluorescent time-lapse microscopy to monitor foci in cells that started budding. To verify the nuclear localization of the RPA foci, we monitored their co-localization with a nuclear signal generated by simultaneous expression of NLS-tetR-tdTomato. Indeed, we found that RPA foci are identified within the cell nucleus following budding and entering S-phase (Fig. S4). Previously, the lack of PCNA ubiquitylation by Rad18 ubiquitin ligase was shown to significantly increase the level of RPA foci in mammalian cells [42]. Thus, to further validate our system, we generated rad18deletion strain and examined the level of RPA foci in mutant cells relative to WT. We found that the percentage of cells with RPA foci containing the rad18-deletion is significantly higher than WT cells (Fig. S5).

Next, we generated the different histone chaperone mutants on the background of GFP-Rfa1 strain and monitored the accumulation of RPA foci in mutant and WT cells (please see Fig. 6A for representative WT and asfl-deleted cells). We found an increase in the percentage of cells containing RPA foci in the histone chaperone mutant cells relative to WT cells indicating that these mutations lead to the accumulation of ssDNA [39,40] (Fig. 6B). Increase in RPA foci was most prominent in asfl- or $r t t 109$-deleted strains, in which over $40 \%$ of the cell population contained at least one clear RPA focus. We found that the increased level of RPA foci in histone chaperone mutant strains is strongly correlated with increased G2/M duration, validating that the delayed G2/M phenotype seen in these strains is related to accumulation of DNA damage during S phase (Fig. S6). Taken together, our results suggest that replication that is uncoupled to nucleosome deposition can lead to replication stress, accumulation of ssDNA, and significant delays in cell cycle progression (Fig. 6 and Fig. S6).

\section{Spontaneous mutation rate analysis of histone chaperone mutant strains}

Replication fork stalling and accumulation of ssDNA during replication can lead to double strand breaks that can be detrimental to cell survival $[44,45]$. To avoid double strand breaks, cells employ a variety of mechanisms including the recruitment of translesion DNA polymerases (TLS) by the $R A D 6 / R A D 18$ pathway [46,47]. This pathway, initiated by mono-ubiquitylation of PCNA on K164, can minimize fork stalling but may lead to increased mutation rates due to the inaccuracy of the TLS polymerases [46,47]. To examine whether mutation rates are elevated in the different histone chaperone mutant strains, we have utilized the CANI assay. We have previously utilized this assay 
to examine a variety of PCNA mutants leading to $R A D 6 / R A D 18$ pathway activation [48]. In accordance with previous analysis, we found that asfl-or rtt109-single deletions lead to a mild increase in mutation rate of up to two-fold relative to the WT strain [49]. Single deletions of $C A C 1$ or RTT106 lead to similarly mild increase. In contrast, we found that the cac1-rtt106-double deletion leads to a dramatic increase of $\sim 7$ fold in mutation rate, (Fig. 7 and Table 1). To examine whether the increased mutation rate in the cacl-rtt106-double deletion strain is due to $R A D 6 / R A D 18$ pathway activation, we generated a cacl-rtt106-rad18-triple deletion strain and examined its mutation rate. Consistent with $R A D 6 / R A D 18$ pathway activation, we observed that the triple deletion strain exhibits a dramatic reduction in mutation rate relative to the cacl-rtt106-double deletion strain (Fig. 7). In addition, we found that depletion of DNA polymerase $\zeta$, a key TLS polymerase, in this strain leads to higher sensitivity to MMS, highlighting its possible activation upon DNA damage (Fig. S7). Taken together, our results with the cac1-rtt106-double deletion strain show that mutations in histone chaperones can lead to $R A D 6 / R A D 18$ pathway activation and increased spontaneous mutation rates. However, results with the other histone chaperone mutant strains, exhibiting long G2/M duration and a mild increase in mutation rate (e.g. rtt109 or asf1 mutant strains), suggest that other pathways can be activated upon ssDNA accumulation during DNA replication (see Discussion section).

\section{Discussion}

DNA RC nucleosome assembly that connects DNA synthesis with nucleosome deposition and modification has been extensively studied in the past two decades $[9,11,50]$. These studies allowed the identification and characterization of histone chaperones that are important for nucleosome deposition on newly synthesized DNA. However, much less is known regarding how mutations in histone chaperones affect replisome progression during DNA replication. Here, we used our recently described direct approach to monitor replisome progression in individual yeast cells $[28,29]$ that are mutated in different histone chaperone genes, including Caf1 subunits, RTT106, ASF1 and RTT109. We found that mutation in Cac1 or Rtt106 that directly deposit H3/H4 histones on DNA, significantly slowdown replication fork progression (Fig. 2-5). These results suggest that the direct interaction of these chaperones with the replication fork is essential for enabling high fork progression rate during S-phase. Interestingly, we found that mutations in histone chaperones which transfer $\mathrm{H} 3 / \mathrm{H} 4$ on Cac1 or Rtt106 prior to their final assembly, including ASF1 or RTT109 histone acetyl transferase, do not affect replisome progression but significantly extend G2/M duration (Fig. 5) highlighting the distinct and independent roles of histone chaperones in genome replication and 
stability. The normal replisome progression in asfl-deleted cells (Fig. 5) is in good agreement with previous studies showing that yeast budding and progression through S-phase is not significantly altered in this mutant strain [20,21].

Our system also allows the detection of elongations in $\mathrm{G} 2 / \mathrm{M}$ duration by monitoring both fluorescence and morphological changes in individual cells. Our findings that single deletion of CAC1, RTT109 or ASF1 or double deletion of CAC1 and RTT106 (Fig. 5) leads to a significant increase in $\mathrm{G} 2 / \mathrm{M}$ duration highlight the important role of these chaperones in preventing replication stress [39]. Our analysis of G2/M duration is probably an underestimation of the true G2/M length in these mutant cells since high percentage of the analyzed cells did not undergo mitosis during the experiment duration (Table S3). Our further examination of these strains revealed a dramatic increase in RPA foci formation following DNA replication (Fig. 6). We found that in the case of double deletion of $C A C 1$ and RTT106 the elongated G2/M duration and RPA foci formation is also correlated with a significant increase in spontaneous mutation rate (Fig. 7). Previously, several studies have extensively examined different aspects of genome stability in histone chaperone mutant strains. An extensive study examining several single and double deletions of histone chaperones including RTT109, ASF1, CAC1 and RTT106 in yeast revealed that most mutations lead to increased recombination frequency, checkpoint activation, loss of replisome stability and sensitivity to DNA damaging agents [14]. In addition, RTT106 was shown to genetically interact with DDC1-MEC3$R A D 17$ that forms an alternative sliding clamp termed the 9-1-1 complex which is involved in checkpoint activation [51]. Furthermore, $A S F 1$ was shown to directly interact with the downstream $R A D 53$ effector kinase $[52,53]$ suggesting that these checkpoint proteins are intimately connected to DNA RC nucleosome deposition. Our findings that histone chaperone mutants lead to high level of RPA foci formation highlight their importance for preventing the accumulation of ssDNA. It complements studies showing the interaction between histone chaperones and S-phase checkpoint activation where ssDNA accumulation sensed by RPA (Fig. 6) is transmitted to several proteins including the 9-1-1 complex [51], leading to Mec1 sensor kinase activation followed by Rad53 effector kinase activation [52,53]. The correlation between high level of RPA foci formation and G2/M duration (Fig. S6) highlights the detrimental effect of ssDNA accumulation for cell cycle progression. In contrast to WT cells in which RPA foci can be corrected during S-phase, we observed that for histone chaperone mutants, RPA foci can be maintained through the G2 phase following DNA replication (Fig. 6A).

Analysis of the different subunits of the Cafl complex indicated that deletion of $C A C 1$ exhibits the strongest effect on both replisome progression and G2/M duration (Fig. 2). Previously, Cac1 has been 
implicated in the MAD2-dependent mitotic spindle checkpoint [36] and our results further verify this study (Fig. 3). Further analysis of mutations in Cac1 WHD or PIP regions showed that while the WHD domain is important for replisome progression, the PIP region is important for preventing replication stress (Fig. 4). This separation of function highlights the importance of direct Cac1-DNA interaction, mediated by the WHD domain, for fast replisome progression. In contrast, elongation of G2/M duration in Cac1 PIP mutant highlights the importance of coordinating Cac1 activity with replisome progression for avoiding replication stress. Our further analysis of the WHD and PIP Cac1 mutants showed a significant reduction of nucleosome deposition of both mutants relative to WT strain as well as slowdown of replisome progression in the presence of HU (Fig. 4 and Fig. S3). Previously, several studies have examined the importance of Cac1 WHD and PIP regions for their biochemical and cellular functions including their importance for DNA binding following association with H3-H4 histones [27], nucleosome assembly in vitro [23] and sensitivity to DNA damaging agents [25]. Our results examining the function of these regions in live cells are in agreement with these studies and further enhance our understanding of the contribution of each interaction for efficient replisome progression and short $\mathrm{G} 2 / \mathrm{M}$ duration.

Previously, mutations in histone chaperones were shown to increase recombination level and gross chromosomal rearrangement highlighting their roles in maintaining genome stability $[14,16]$. Asfl, Caf1 and Rtt109 were shown to be important for deactivation of damage checkpoint after double strand break repair [55,56]. However, much less is known regarding their contribution to DNA damage response pathways that include the activation of TLS polymerases for damage bypass. Our findings that mutations in histone chaperones lead to moderate increase in spontaneous mutation rate (Fig. 7) suggest that other pathways are dominant, including homologues recombination, to overcome replication stress. Our findings are in good correlation with a previous study examining the level of spontaneous mutation rate in several deletion strains including the RTT109 or ASF1 single deletions [49]. The significant increase in spontaneous mutation rate in cells deleted in CAC1 and RTT106 (Fig. 7) may suggest that these chaperones link PCNA both to nucleosome deposition and activation of TLS polymerases through PCNA ubiquitylation by the RAD6/RAD 18 pathway. Notably, these results are in agreement with the involvement of $C A C 1$ in the $R A D 6 / 18$ post-replication repair pathway [32].

Overall, our experiments reveal that histone chaperones exhibit distinct functions in replisome progression and maintaining genome stability. Our data showing the effects of cacl-or $r t t 106$-deletion on replisome progression highlight the possible importance of direct interaction of these chaperones with $\mathrm{H} 3 / \mathrm{H} 4$ and the DNA in facilitating fast replisome progression. In contrast, histone chaperones which are involved indirectly with the transfer of histones on DNA, including Asf1 and Rtt109 do not 
significantly affect replisome progression but are essential for preventing replication stress. Our system can be further utilized to investigate how additional histone chaperones, such as the FACT complex, Nap1, Vps75 [5,57], or combinations of mutants affect replication fork progression and G2/M duration.

\section{Materials and Methods}

\section{Strain generation}

Strains for replication time measurement were generated on the background of a W303 MATa Saccharomyces cerevisiae strain, expressing GFP-LacI and tetR-tdTomato fusion proteins in the nucleus. lacOx256 and tetOx224 arrays are located at chrIV:332960 and chrIV:352560 respectively, near ARS413 and with a mid-array distance of approximately $30.6 \mathrm{~kb}$ [28]. CAC1, CAC2, CAC3, RTT106, ASF1, RTT109 and MAD2 genes were replaced by natMX or hphMX antibiotic cassettes using the Lithium Acetate (LiAc) transformation method [58]. The cac1 WHD, cac1 PIP, and rtt106 KK245,246AA mutants were generated on the background of the cacl- or rtt106- deleted cells respectively, with a markerless CRISPR/Cas9 approach, by targeting either the natMX or hphMX with specific gRNAs and the respective mutant genes as DNA donors [59]. All replacements were validated with Polymerase Chain Reaction (PCR) and Sanger sequencing.

\section{Microscopy and data analysis}

All microscope experiments for the determination of replication time were conducted as previously described [28] with slight modifications. Briefly, yeast cultures were grown overnight to $\mathrm{OD}_{600 \mathrm{~nm}}=$ 0.1-0.2 in synthetic complete (SC) medium containing $4 \%$ glucose at $30^{\circ} \mathrm{C}$. The cultures were then synchronized at G1 using $10 \mu \mathrm{g} / \mathrm{ml} \alpha$-factor (GenScript) for 2-3 hours. For hydroxyurea (HU) experiments, yeast cultures were incubated with $20 \mathrm{mM} \mathrm{HU}$ one hour before and during the imaging. Cells were then immobilized on microscopy slide chambers (Ibidi) coated with $2 \mathrm{mg} / \mathrm{ml}$ concanavalin A (Sigma-Aldrich) and washed thoroughly from $\alpha$-factor with warm SC medium containing 4\% glucose prior to microscopy measurements. Live cell imaging of the cells was performed on a Marianas spinning-disk confocal microscope (3i) using an Evolve EM-CCD camera (Photometrics) with 1 min intervals for $2-8$ hours at $28^{\circ} \mathrm{C}$, using a x63 oil objective $(\mathrm{NA}=1.4)$ in 3D (12 z-sections at $0.7 \mu \mathrm{m}$ apart). GFP-LacI and TetR-tdTomato were excited with $488 \mathrm{~nm}$ and $561 \mathrm{~nm}$ lasers, respectively. For data analysis, time-lapse measurements were collected with SlideBook (3i) and analyzed in Matlab using a custom-made software ('DotQuant') which identifies, tracks and quantifies the fluorescent dots in each cell [28]. Statistical analysis of 
replication rate results was performed using Monte Carlo resampling with 1,000,000 iterations. Mitosis events were identified manually as the timepoint when the dots split and move to the mother and daughter cell.

\section{Chromatin immunoprecipitation (ChIP) assay}

ChIP assays were performed using anti-H3K56Ac antibodies as previously described [19]. Exponentially growing cells were synchronized with $5 \mu \mathrm{g} / \mathrm{ml} \alpha$-factor for $3 \mathrm{hr}$ at $25^{\circ} \mathrm{C}$ and then released into fresh media containing $0.2 \mathrm{M} \mathrm{HU}$ for $45 \mathrm{~min}$ at $25^{\circ} \mathrm{C}$. Samples were collected to perform ChIP assay using H3K56Ac and H3 (as a normalization control) antibodies. The ChIP DNA was analyzed by quantitative PCR using primers (ARS607/ARS607+14kb and $A R S 305 / A R S 305+12 \mathrm{~kb}$ ) amplifying replication origins as well as fragments downstream of replication origins. The mean and SD of three independent biological replicates were shown. The percentage of ChIP DNA relative to the total input DNA was calculated (Fig. 4), and the ratio of H3K56Ac ChIP signal to the total H3ChIP signal was also calculated (Fig. S2).

\section{RPA foci analysis}

Strains for RFA1 foci analysis were generated on the background of a BY4741 Saccharomyces cerevisiae strain which carries N-terminus GFP-tagged RFA1 expressed under control of its native promoter [43], as described above. To locate the cell nuclei, NLS-tetR-tdTomato was transformed into the ADE1 locus. For imaging, yeast cultures were grown overnight at $30^{\circ} \mathrm{C}$ to $\mathrm{OD}_{600 \mathrm{~nm}}=0.2$ 0.6 in SC medium containing 4\% glucose. Cells were fixed on microscopy slide chambers (Ibidi) coated with $2 \mathrm{mg} / \mathrm{ml}$ concanavalin A (Sigma-Aldrich) and imaged using confocal microscopy as described above for $1-3 \mathrm{~h}$ with $1 \mathrm{~min}$ intervals at $28^{\circ} \mathrm{C}$. GFP-Rfal and NLS-tetR-tdTomato were excited with $488 \mathrm{~nm}$ and $561 \mathrm{~nm}$ lasers, respectively. At least 60 cells from three independent experiments were analysed for each strain and scored for the presence of one or more RPA foci during S-phase. The percentage of cells with RPA foci was estimated by dividing the number of cells exhibiting one or more RPA foci in the nucleus by the total amount of cells that were taken into account in each experiment. Results were compared with one-way ANOVA and post-hoc analysis (Dunnett's test), after transforming the data with $\arcsin (\operatorname{sqrt}(\mathrm{x}))$.

\section{Can1 spontaneous mutation rate analysis}

The determination of the mutation rate of the canl gene was performed as previously described [60] based on the Lea and Coulson method [61] with some modifications. Strains for canl mutation rate measurement were generated on the background of a BY4741 Saccharomyces cerevisiae strain as 
described above. Yeast cultures were grown overnight at $30^{\circ} \mathrm{C}$ in YPD medium to $\mathrm{OD}_{600 \mathrm{~nm}} \approx 0.8-1$. Cells were then diluted $10^{5}$ fold, plated as single cells on SC plates and grown for 4 days at $30^{\circ} \mathrm{C}$. At least 16 single colonies were randomly chosen from each strain, resuspended in $1 \mathrm{ml}$ of sterile $\mathrm{ddH}_{2} \mathrm{O}$ and diluted to $\mathrm{OD}_{600 \mathrm{~nm}} \approx 1.0$. Next, $10^{5}$ fold dilutions of each resuspended colony solution were plated on SC plates to define the number of viable cells and the rest of the solutions were plated on SC-Arg $+60 \mathrm{mg} / \mathrm{ml}$ canavanine to obtain the number of canavanine-resistant cells. The median number of viable cells was estimated from the different colonies (replicates) and the mutation rates, the $95 \%$ Confidence Intervals (CI) and the $p$ values were calculated by utilising the rSalvador package (v1.7) running on the R software (v3.4.3) [62].

\section{Rev3 inducible degradation and spot assays}

Strains for spot assays were generated on the background of a BY4741 Saccharomyces cerevisiae strain. An auxin-inducible degron (AID) was inserted into the C'-terminus of rev3 as previously described [28]. Briefly, a PCR fragment containing the AID sequence, the Oryza sativa TIR1 gene and the $h p h M X$ resistance cassette as selection marker was generated with homologous ends to the $5^{\prime}$ of the $R E V 3$ yeast gene. The presence of AID results in the degradation of the fused protein after incubation of yeast cells with 1-Napthaleneacetic acid (NAA) [63,64]. The viability of rev3-AID histone-chaperone mutants was tested with spot assays on agarose plates. Briefly, overnight exponentially-growing yeast cultures were diluted to $\mathrm{OD}_{600 \mathrm{~nm}}=0.3$ and $4 \mu 1$ of 10 -fold serial dilutions were spotted on YPD plates containing 200 $\mu \mathrm{g} / \mathrm{ml} \mathrm{HYG}, \mathrm{HYG}+0.5 \mathrm{mM}$ NAA, $\mathrm{HYG}+0.005 \% \mathrm{MMS}$, or $\mathrm{HYG}+0.5 \mathrm{mM}$ NAA $+0.005 \%$ MMS. Cells were incubated at $30^{\circ} \mathrm{C}$ for 32 hours and representative pictures were captured. 


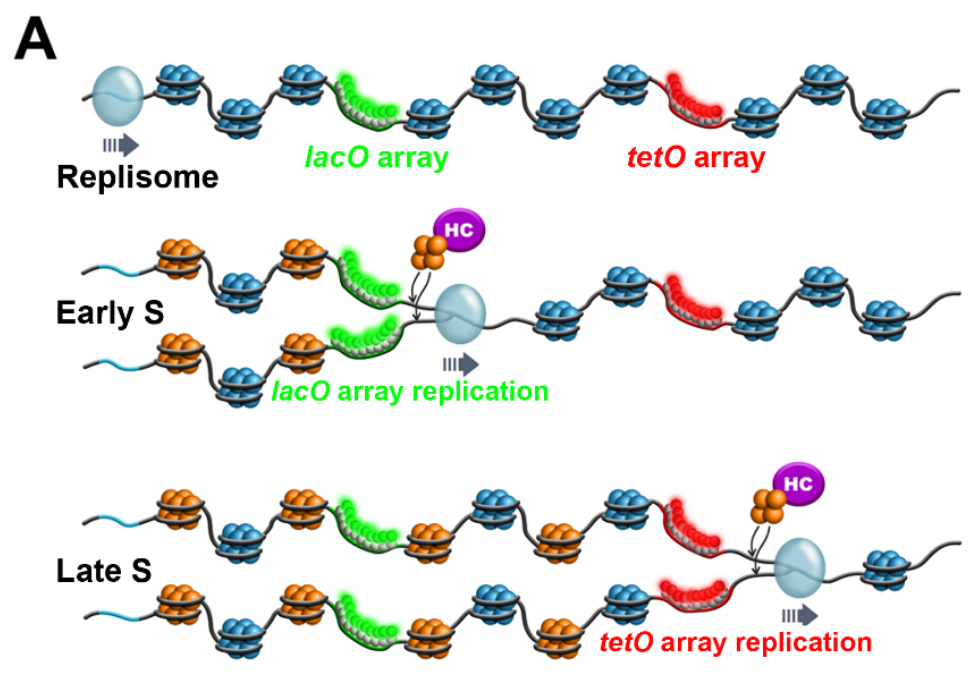

B

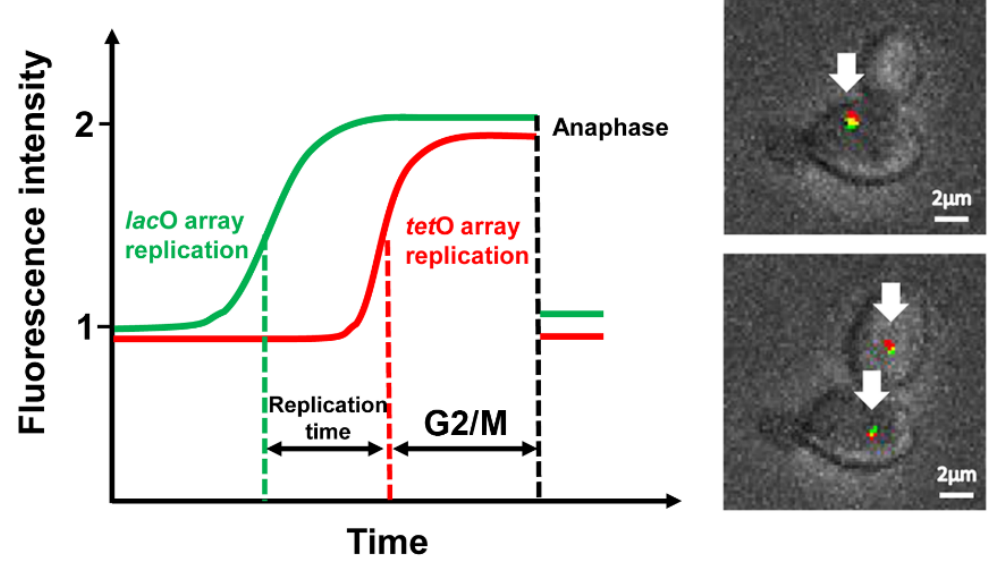

Figure 1: Measuring replication rates in histone chaperone mutant yeast cells. (A) Schematic illustration of the design for the real-time analysis of replication kinetics in live yeast cells containing mutations in histone chaperone genes. Strains are genetically engineered to contain $l a c O$ and tet $O$ arrays located $20 \mathrm{~Kb}$ apart, downstream from an origin of replication. Binding of GFP-lacI and tetR-tdTomato lead to green and red fluorescent foci, respectively. During DNA replication, array duplication leads to recruitment of additional lacI-GFP and tetR-tdTomato proteins leading to an increase in fluorescence intensity. The newly-synthesized DNA is packed around new (orange) and pre-existing (blue) nucleosomes. Histone chaperones (HC, purple) deposit newly-synthesized histones as $(\mathrm{H} 3 / \mathrm{H} 4)_{2}$ tetramers behind the replisome. The replication time of each locus is measured using time-lapse confocal microscopy. For simplicity, origin firing is shown only to the array direction. (B) Schematic display of the doubling of fluorescent intensity of the GFP-lacI and tetR-tdTomato foci in a single cell due to $l a c O$ and tet $O$ array duplication during DNA replication. The time between replication of the arrays (replication time) is calculated using the mid-rise points of the GFP and tdTomato fluorescence intensities. The G2/M duration is estimated by the time delay between the mid-rise point of the tdTomato fluorescence intensity and the anaphase $(\mathbf{C})$ Image of single cells from the yeast strains used in this study. Top - image following replication before anaphase, Bottom - after anaphase. Images scale: $2 \mu \mathrm{m}$ 

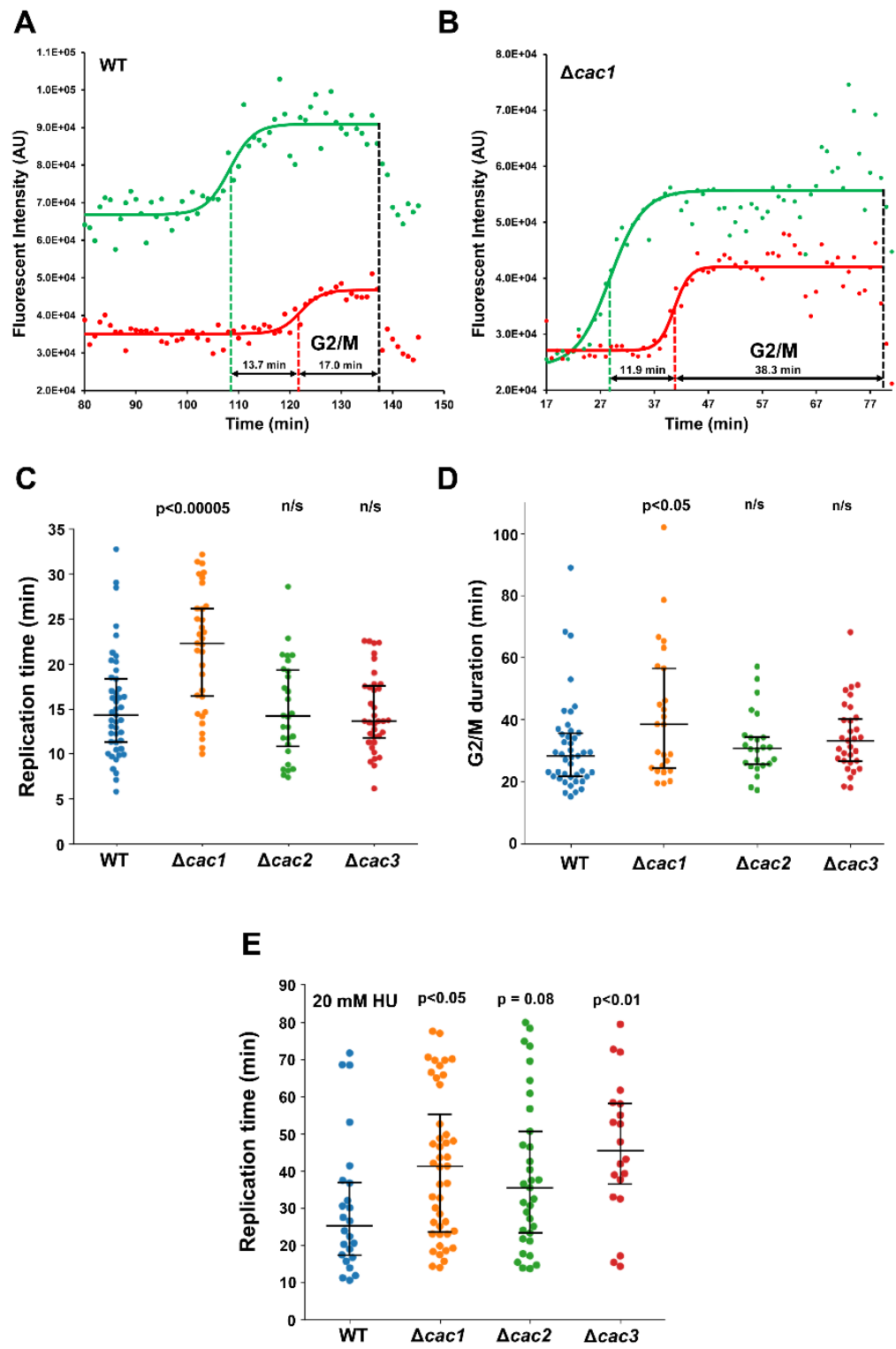

Figure 2: Extended replication times and G2/M duration in cac1-deleted cells. (A-B) Representative results of a single cell from (A) WT and (B) cacl-deleted strains showing a significant increase in G2/M duration in the cacl-deleted cell. Solid lines represent a fit of the data to a sigmoidal function showing the $\mathrm{lac} O$ array replication (green) and the tet $O$ array replication (red), green and red mid-points are indicated by dashed lines while black dashed lines show the anaphase timepoint. Black double arrows measuring the time delay between lac $O$ array replication and tet $O$ array replication and tet $O$ array replication and anaphase indicates the replication time and G/M duration, respectively. (C) Replication times of $\sim 30.6$ $\mathrm{Kb}$ at the vicinity of ARS413 for WT, cac1-, cac2- and cac3-deleted cells. (D) G2/M durations, estimated by measuring the time delay between the mid-rise point of the tdTomato fluorescence intensity and the anaphase, for WT, cac1-, cac2- and cac3-deleted cells. (E) Replications times as in (C) but in the presence of $20 \mathrm{mM}$ of HU. Significance was determined by Monte Carlo resampling and $p$ values relative to WT are shown. The number of cells analyzed for each strain, all median values and statistical results are shown in Table S1 and for (E) Table S2. 

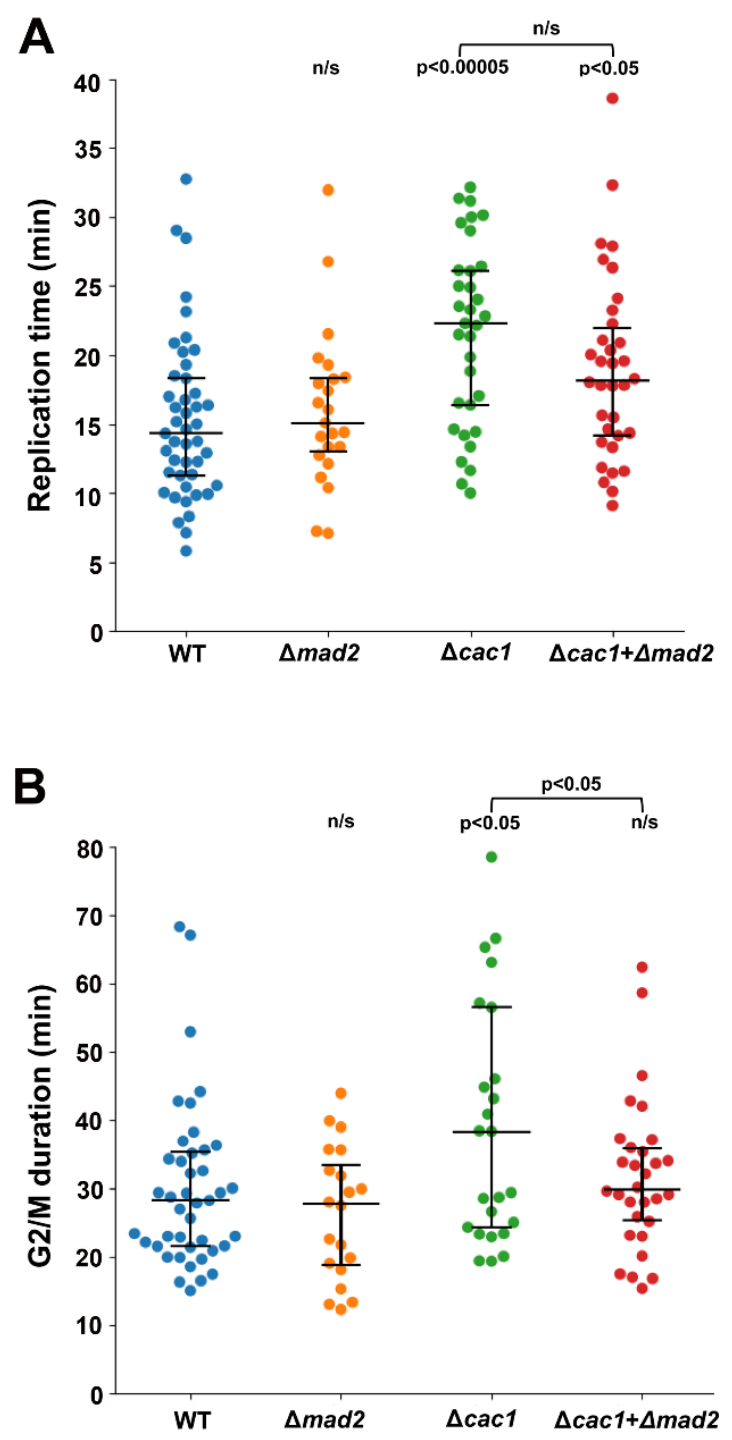

Figure 3: Extended G2/M duration in cac1-deleted cells is suppressed by the deletion of MAD2: (A) Replication times of $\sim 30.6 \mathrm{~Kb}$ at the vicinity of ARS413 for WT, mad2-, cacldeletion and mad2 and cacl-double deleted cells. (B) G2/M durations, estimated by measuring the time delay between the mid-rise point of the tdTomato fluorescence intensity and the anaphase, for WT, mad2-, cac1-deletion and mad2 and cacl-double deleted cells. The number of cells analyzed for each strain and all median values and statistical results are shown in Table $\mathbf{S 1}$. 

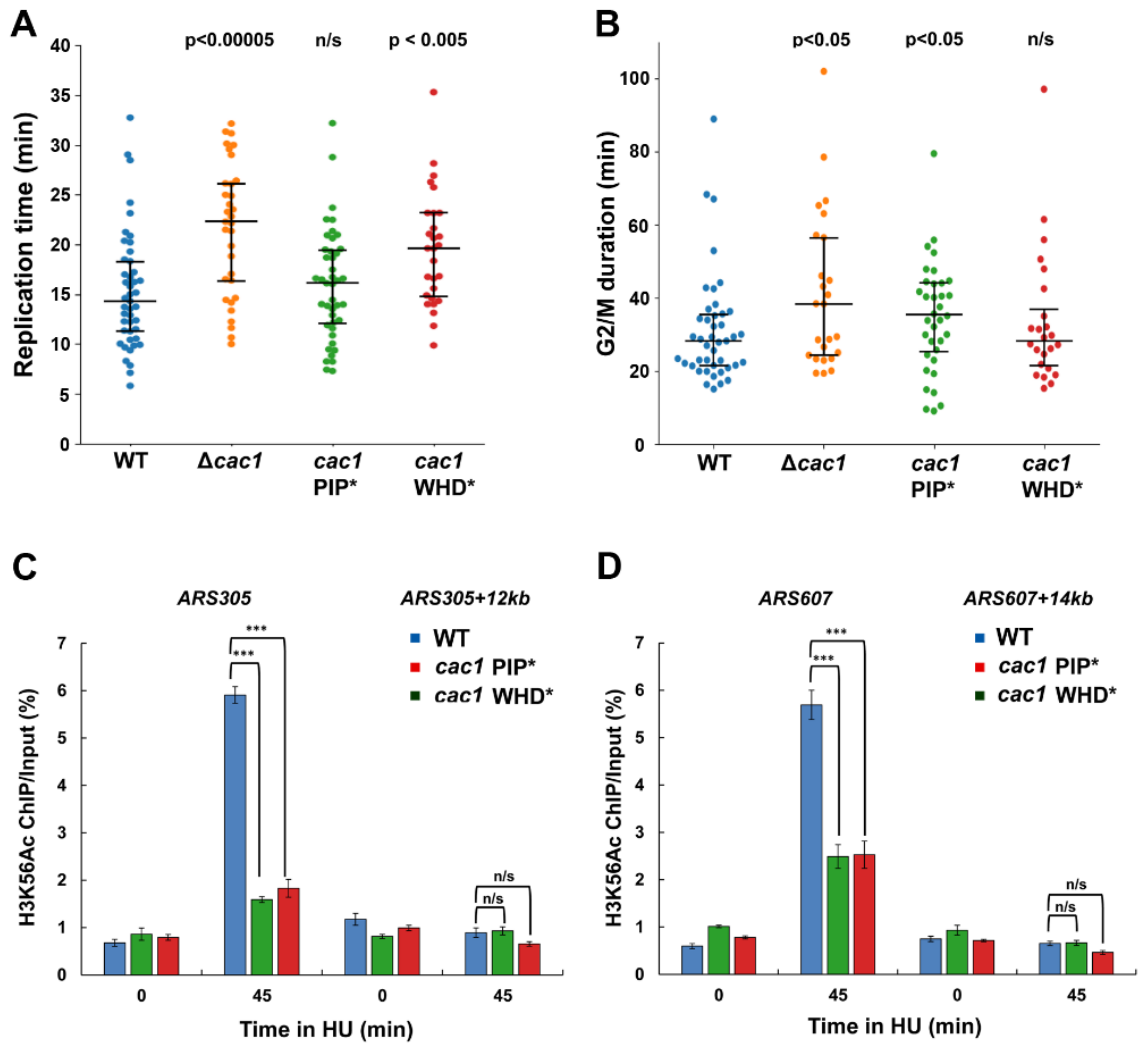

Figure 4: Replication times, G2/M duration and ChIP analysis of cac1 mutated cells. Mutations in the Cac1 WHD an PIP domains lead to increased replication times and G2/M duration, respectively, and lower nucleosome deposition during DNA replication, relative to WT cells. (A) Replication times of $\sim 30.6 \mathrm{~Kb}$ at the vicinity of ARS413 for WT, cacldeletion, cac1 PIP* (PIP mutant containing the F233A and F234G mutations)- and cac1 WHD* (WHD mutant containing the K564E and K568E mutations)-mutant cells. (B) G2/M durations, calculated as described in Fig. 2D, for WT, cacl-deleltion, cacl PIP*- and cacl WHD-mutant cells. Significance was determined by Monte Carlo resampling and $p$ values relative to WT are shown. The number of cells analyzed for each strain and all median values and statistical results are shown in Table S1. (C-D). ChIP analysis of H3K56Ac deposition for WT and cacl mutant strains. WT and cacl mutant cells were arrested at G1 phase using $\alpha$-factor at $25^{\circ} \mathrm{C}$ and then released into fresh medium containing $0.2 \mathrm{M} \mathrm{HU}$. Equal numbers of cells were collected just prior to ( $\mathrm{G} 1,0 \mathrm{~min})$ and at 45 minutes following release into fresh medium containing HU. ChIP assays were performed using antibodies against H3K56Ac and $\mathrm{H} 3$ (as a normalization control). The ChIP DNAs were analyzed using four different primer pairs amplifying (C) $A R S 305, A R S 305+12 \mathrm{~kb}$ and (D) $A R S 607$, and $A R S 607+14 \mathrm{~kb}$, using quantitative PCR. At least three independent biological repeats were performed, with similar trends in all cases. The mean and SDs of three independent biological replicates are shown. $P$ values derived from two-way analysis of variance (ANOVA) $(* * * P$ value $\leq 0.001)$. ChIP over input is shown here, results were similar after normalizing to total $\mathrm{H} 3 \mathrm{ChIP}$ for the same trend (Fig. S2), indicating that loss of H3K56Ac signal is not as a result of decreased total H3. 

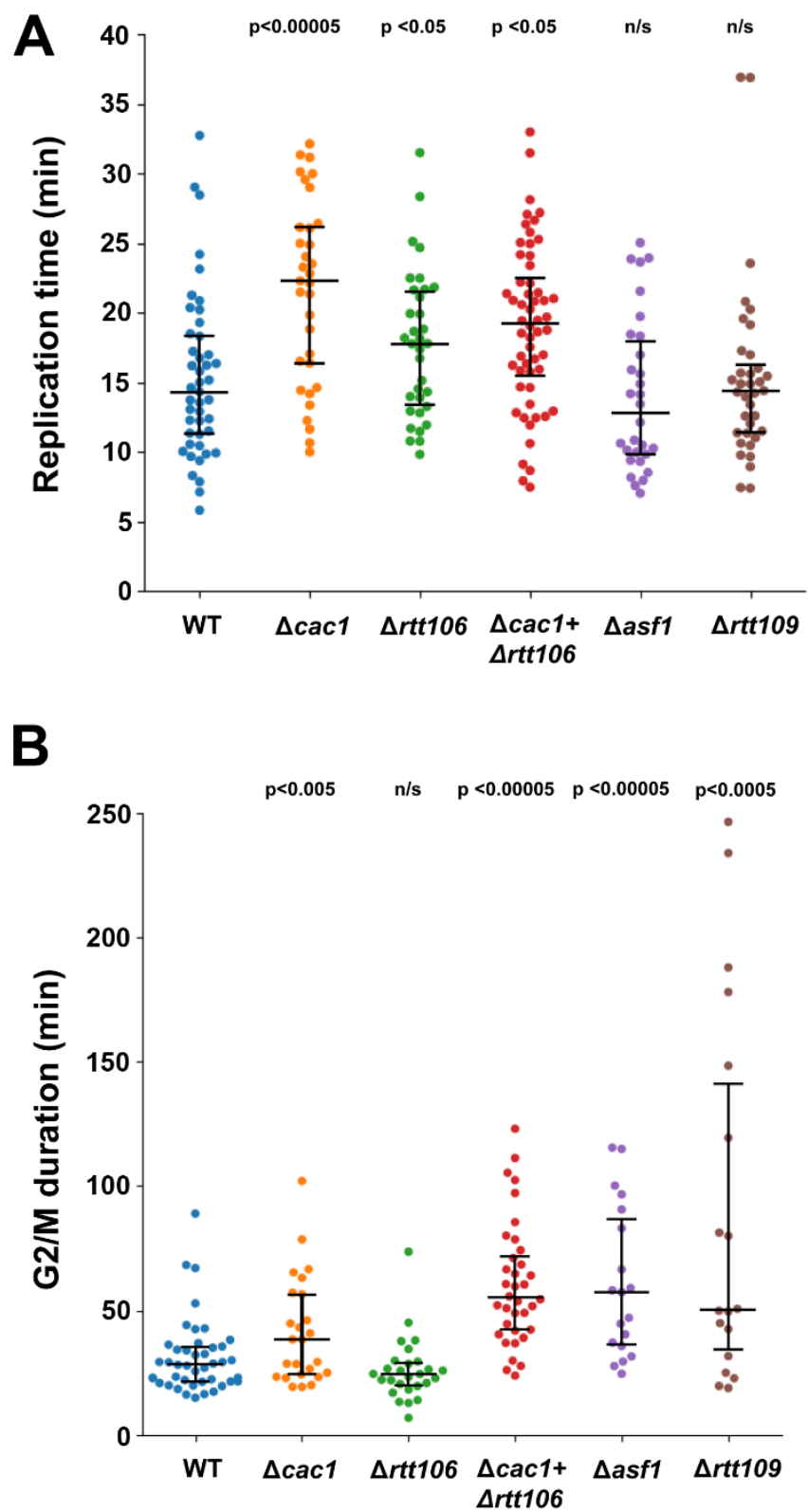

Figure 5: Replication times and G2/M durations of cac1-, rtt106-, cac1- and rtt106-, asf1or rtt109-deleted cells. Cell measurements are shown as swarm plots (A) Replication times of $\sim 30.6 \mathrm{~Kb}$ at the vicinity of ARS413 for WT, cacl-, rtt106-, cacl- and rtt106-, asfl- or rtt109-deleted cells. WT and cacl-deleted cells are shown as comparison. (B) G2/M durations, estimated by measuring the time delay between the mid-rise point of the tdTomato fluorescence intensity and the anaphase, for WT, cacl-, rtt106-,cacl- and rtt106-, asfl- or rtt109-deleted cells. Significance was determined by Monte Carlo resampling and $\mathrm{p}$ values relative to WT are shown. The number of cells analyzed for each strain and all median values and statistical results are shown in Table $\mathbf{S 1}$. 


\section{A \\ WT}

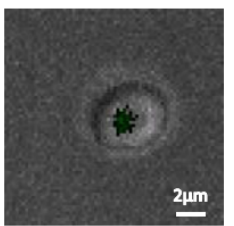

$\mathrm{t}=36 \mathrm{~min}$

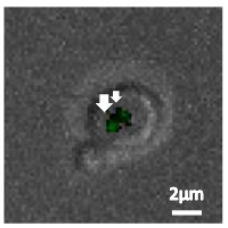

$\mathbf{t}=59 \min$

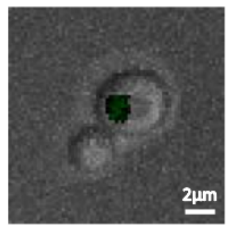

$\mathrm{t}=78 \mathrm{~min}$

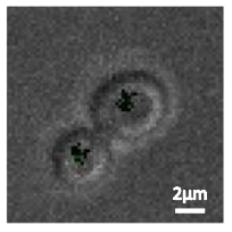

$t=114 \min$

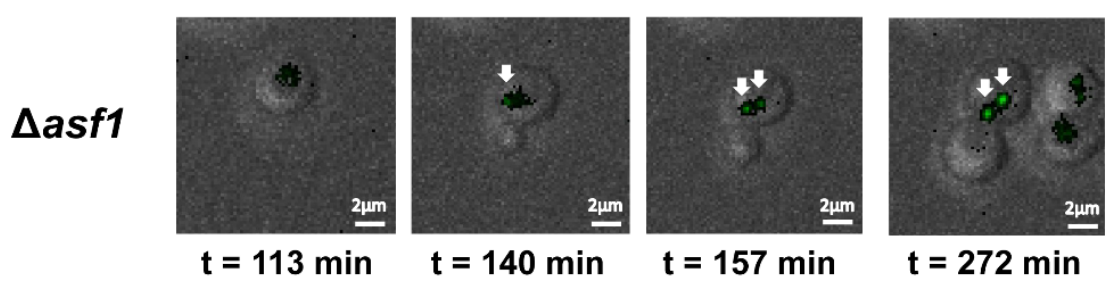

B

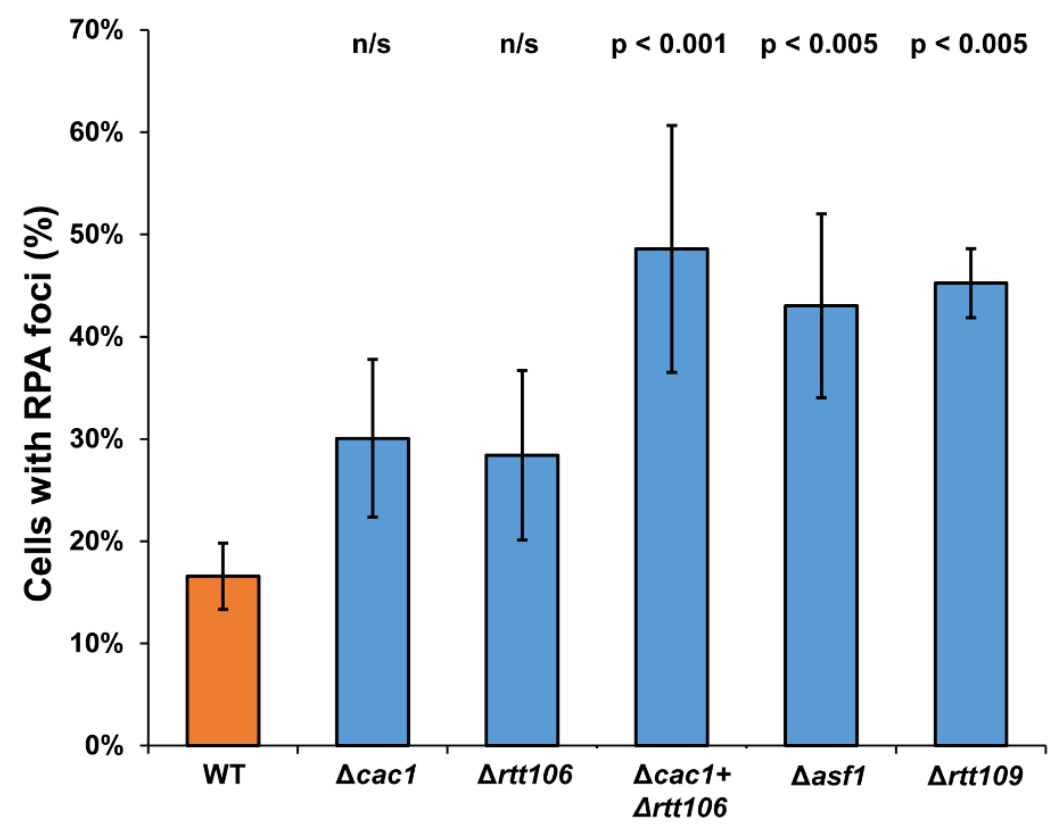

Figure 6: RPA foci accumulate in histone chaperone mutant cells. (A) Representative WT and asf1-deleted cells containing RPA foci are shown at different time points. In the case of the WT cells, the RPA focus disappears in late S-phase. In the case of asfl-deleted cell, mitosis does not take place even after $120 \mathrm{~min}$ from initial budding and the RPA foci persists. (B) WT or histone chaperone mutant cells expressing N-terminal GFP-Rfal fusion were analyzed using time-lapse fluorescent microscopy. At least 60 cells from three independent experiments were analyzed for each strain and scored for the presence of one or more RPA foci during S-phase. Images scale: $2 \mu \mathrm{m}$ 


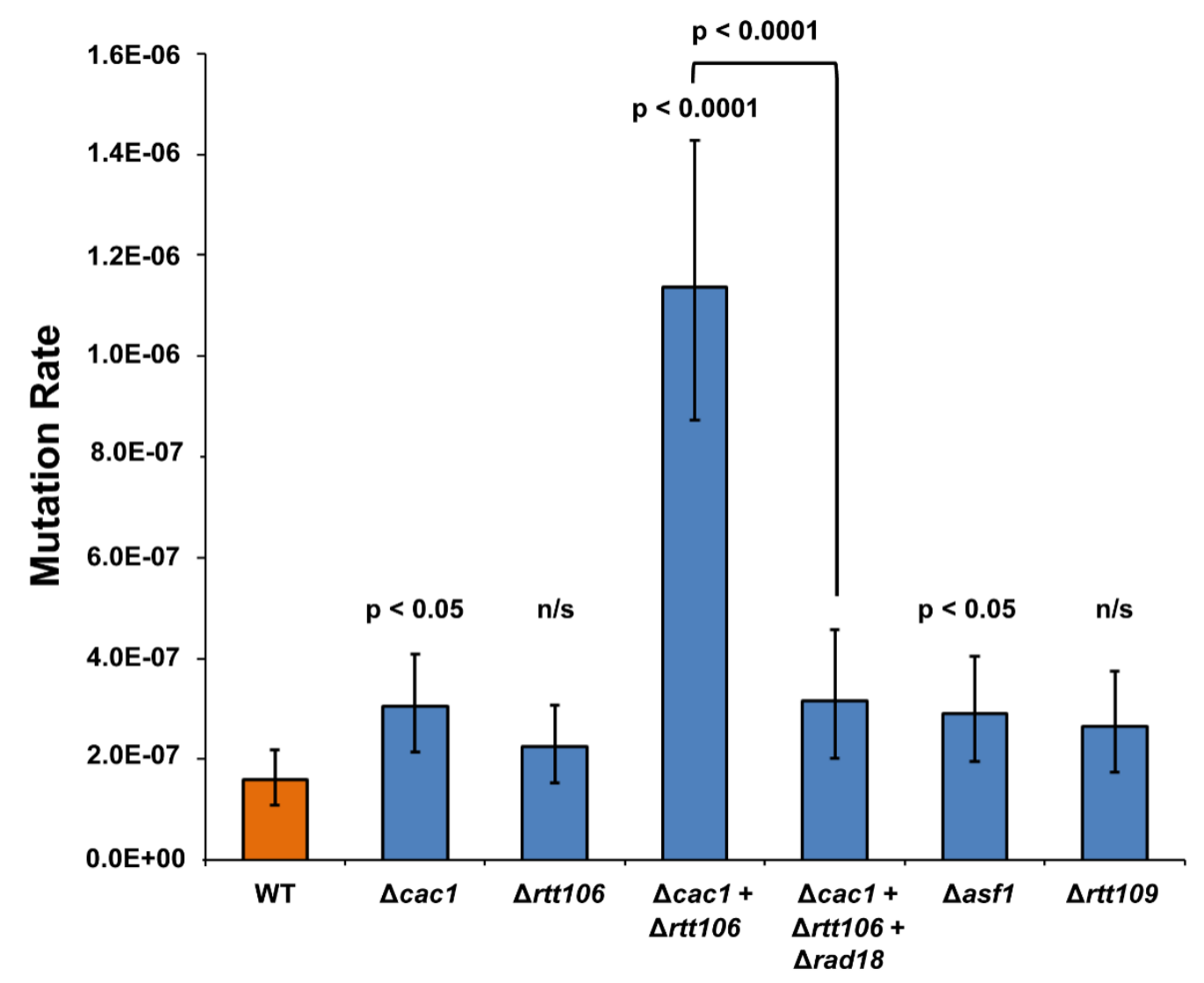

Figure 7: Increased spontaneous mutation rate in histone chaperone mutants is most significant in the cac1-rtt106-double deletion strain. Spontaneous mutations in this strain are dependent upon $R A D 6 / R A D 18$ pathway since spontaneous mutation rate in the triple cac1-rtt106-rad18-deletion strain is dramatically reduced relative to the cac1-rtt106-double deletion strain. The data are rates of $\mathrm{Can}^{\mathrm{r}}$ mutation values within the $95 \%$ confidence limits of at least 16 independent analyses. The values of the spontaneous mutation rate of the different strains are presented in Table 1. 
Table 1: Spontaneous mutation rate at the CAN1 locus

\begin{tabular}{|l|c|c|}
\hline Genotype & Mutation rate Can ${ }^{\mathbf{R}}[\mathbf{C I}]^{\mathrm{a}}$ & $\begin{array}{c}\text { Fold increase } \\
\text { compared to } \\
\text { WT }\end{array}$ \\
\hline $\mathrm{WT}$ & $1.59[1.09,2.19] \times 10^{-7}$ & - \\
\hline$\Delta c a c 1$ & $3.04[2.14,4.08] \times 10^{-7}$ & 1.9 \\
\hline$\Delta r t t 106$ & $2.24[1.54,3.08] \times 10^{-7}$ & $1.4^{\mathrm{b}}$ \\
\hline$\Delta c a c 1+\Delta r t t 106$ & $11.4[8.72,14.30] \times 10^{-7}$ & 7.2 \\
\hline$\Delta c a c 1+\Delta r t t 106+\Delta \mathrm{rad} 18$ & $3.15[2.01,4.57] \times 10^{-7}$ & 2.0 \\
\hline$\Delta a s f 1$ & $2.90[1.95,4.05] \times 10^{-7}$ & 1.8 \\
\hline$\Delta r t t 109$ & $2.64[1.75,3.74] \times 10^{-7}$ & $1.7^{\mathrm{b}}$ \\
\hline
\end{tabular}

${ }^{a}$ The numbers in brackets represent the low and high values for the 95\% confidence interval for each rate, obtained using the confidence interval for the median test. The medians and 95\% confidence intervals were deduced from at least 16 independent determinations for each strain.

${ }^{b}$ Non significant difference from the WT 
Figure S1: Growth defects of cac1-deletion strain is suppressed by mad2-deletion.

Growth was analyzed by a spot assay on YPD plates using a 10-fold serial dilution of yeast cultures spotted on the plate. Growth was analyzed following $24 \mathrm{~h}$ (left) or 48h (right) of incubation.

Figure S2: The Cac1 PIP*(F233AF234G) or Cac1 WHD*(K564EK568E) mutations lead to reduced new H3 deposition on replicating DNA. Results are normalized to total H3 ChIP, complementing data shown in Figure 3 (main text) indicating that loss of H3K56Ac signal is not as a result of decreased total H3.

Figure S3: Replication times of cac1 mutated cells measure in the presence of $20 \mathrm{nM}$ of UH. Mutations in the Cac1 WHD an PIP domains lead to increased replication times relative to WT cells in the presence of HU. Replication times of $\sim 30.6 \mathrm{~Kb}$ at the vicinity of ARS413 for WT, cac1-deleltion, cac1 PIP*(PIP mutant containing the F233A and F234G mutations)and cacl WHD* (WHD mutant containing the K564E and K568E mutations)-mutant cells. The number of cells analyzed for each strain and all median values and statistical results are shown in Table S2.

Figure S4: Expression of RFA1-GFP and NLS-tetR-tdTomato for validation that RPA foci are located in the cell nucleus. Representative WT and asfl-deleted cells containing RPA foci are shown.

Figure S5: The percentage of cells with RPA foci is significantly increased in $\Delta$ rad18 cells relative to WT. Analysis of RPA foci is described in the Materials and Methods section and in Figure 6 of the main text.

Figure S6: Pearson correlation between percentage of cells with RPA foci and G2/M duration. the $\mathrm{p}$ value is 0.017 indicating a statistically significant correlation.

Figure S7: Depletion of DNA polymerase $\zeta$ in cac1- and rtt106-double deleted strain leads to higher sensitivity to DNA damage. All analysed yeast strains contain REV3 (the catalytic subunit of DNA polymerase $\zeta$ ) fused to auxin inducable degron (AID) to enable its depeletion following NAA (auxin) addition to the growth media (see material and methods for details). Growth of WT, single cacl-or rtt106-deletion strains and the double cacl-and rtt106-deletion strains on plates containing YPD or YPD supplemented with $0.005 \%$ MMS were analysed with or without NAA. 
Table S1: Parameters obtained from live cell microscopy analysis of different histone chaperone mutant strains described in the main text.

Table S2: Parameters obtained from live cell microscopy analysis described in the main text of different histone chaperone mutant strains in the presence of HU.

Table S3: Percentage of cells undergoing mitosis at a time duration of up to 90 minutes following replication of the tetR labeled array. 


\section{References}

1. Alabert C, Groth A. Chromatin replication and epigenome maintenance. Nat Rev Mol Cell Biol. 2012;13: 153-167. doi:10.1038/nrm3288

2. Papamichos-Chronakis M, Peterson CL. Chromatin and the genome integrity network. Nat Rev Genet. 2012;14: 62-75. doi:10.1038/nrg3345

3. Andrews AJ, Luger K. Nucleosome Structure(s) and Stability: Variations on a Theme. Annu Rev Biophys. 2011;40: 99-117. doi:10.1146/annurev-biophys042910-155329

4. Falbo KB, Shen X. Chromatin remodeling in DNA replication. J Cell Biochem. 2006;97: 684-689. doi:10.1002/jcb.20752

5. Hammond CM, Strømme CB, Huang H, Patel DJ, Groth A. Histone chaperone networks shaping chromatin function. Nat Rev Mol Cell Biol. 2017;18: 141-158. doi:10.1038/nrm.2016.159

6. Mattiroli F, D'Arcy S, Luger K. The right place at the right time: chaperoning core histone variants. EMBO Rep. 2015;16: 1454-1466. doi:10.15252/embr.201540840

7. Gurard-Levin ZA, Quivy J-P, Almouzni G. Histone chaperones: assisting histone traffic and nucleosome dynamics. Annu Rev Biochem. 2014;83: 487-517. doi:10.1146/annurev-biochem-060713-035536

8. Li Q, Burgess R, Zhang Z. All roads lead to chromatin: Multiple pathways for histone deposition. Biochim Biophys Acta - Gene Regul Mech. 2012;1819: 238246. doi:10.1016/j.bbagrm.2011.06.013

9. Serra-Cardona A, Zhang Z. Replication-Coupled Nucleosome Assembly in the Passage of Epigenetic Information and Cell Identity. Trends Biochem Sci. 2018;43: 136-148. doi:10.1016/j.tibs.2017.12.003

10. Wellinger RE, Prado F, Aguilera A. Replication Fork Progression Is Impaired by Transcription in Hyperrecombinant Yeast Cells Lacking a Functional THO Complex Replication Fork Progression Is Impaired by Transcription in Hyperrecombinant Yeast Cells Lacking a Functional THO Complex. 2006;26: 3327-3334. doi:10.1128/MCB.26.8.3327 
11. Prado F, Maya D. Regulation of replication fork advance and stability by nucleosome assembly. Genes (Basel). 2017;8. doi:10.3390/genes8020049

12. Zhang L, Serra-Cardona A, Zhou H, Wang M, Yang N, Zhang Z, et al. Multisite Substrate Recognition in Asf1-Dependent Acetylation of Histone H3 K56 by Rtt109. Cell. 2018;174: 818-830.e11. doi:10.1016/j.cell.2018.07.005

13. Panne D, Sauer P V., Gu Y, Liu WH, Mattiroli F, Luger K, et al. Mechanistic insights into histone deposition and nucleosome assembly by the chromatin assembly factor-1. Nucleic Acids Res. 2018;46: 9907-9917. doi:10.1093/nar/gky823

14. Clemente-Ruiz M, González-Prieto R, Prado F. Histone H3K56 acetylation, CAF1, and Rtt106 coordinate nucleosome assembly and stability of advancing replication forks. PLoS Genet. 2011;7. doi:10.1371/journal.pgen.1002376

15. Zunder RM, Antczak AJ, Berger JM, Rine J. Two surfaces on the histone chaperone Rtt106 mediate histone binding, replication, and silencing. Proc Natl Acad Sci. 2011;109: E144-E153. doi:10.1073/pnas.1119095109

16. Myung K, Pennaneach V, Kats ES, Kolodner RD. Saccharomyces cerevisiae chromatin-assembly factors that act during DNA replication function in the maintenance of genome stability. Proc Natl Acad Sci. 2003;100: 6640-6645. doi:10.1073/pnas.1232239100

17. Yang J, Zhang X, Feng J, Leng H, Li S, Xiao J, et al. The Histone Chaperone FACT Contributes to DNA Replication-Coupled Nucleosome Assembly. Cell Rep. 2016;14: 1128-1141. doi:10.1016/j.celrep.2015.12.096

18. Mejlvang J, Feng Y, Alabert C, Neelsen KJ, Jasencakova Z, Zhao X, et al. New histone supply regulates replication fork speed and PCNA unloading. J Cell Biol. 2014;204: 29-43. doi:10.1083/jcb.201305017

19. Li Q, Zhou H, Wurtele H, Davies B, Horazdovsky B, Verreault A, et al. Acetylation of Histone H3 Lysine 56 Regulates Replication-Coupled Nucleosome Assembly. Cell. 2008;134: 244-255. doi:10.1016/j.cell.2008.06.018

20. Ramey CJ, Howar S, Adkins M, Linger J, Spicer J, Tyler JK. Activation of the DNA Damage Checkpoint in Yeast Lacking the Histone Chaperone Anti-Silencing 
Function 1. Mol Cell Biol. 2004;24: 10313-10327. doi:10.1128/mcb.24.23.1031310327.2004

21. Kats ES, Albuquerque CP, Zhou H, Kolodner RD. Checkpoint functions are required for normal S-phase progression in Saccharomyces cerevisiae RCAF- and CAF-Idefective mutants. Proc Natl Acad Sci. 2006;103: 3710-3715. doi:10.1073/pnas.0511102103

22. Hoek M, Stillman B. Chromatin assembly factor 1 is essential and couples chromatin assembly to DNA replication in vivo. Proc Natl Acad Sci. 2003;100: 12183-12188. doi:10.1073/pnas.1635158100

23. Krawitz DC, Kama T, Kaufman PD. Chromatin assembly factor I mutants defective for PCNA binding require Asf1/Hir proteins for silencing. Mol Cell Biol. 2002;22: 614-25. Available: http://www.ncbi.nlm.nih.gov/pubmed/11756556

24. Moldovan G-L, Pfander B, Jentsch S. PCNA, the Maestro of the Replication Fork. Cell. 2007;129: 665-679. doi:10.1016/j.cell.2007.05.003

25. Zhang K, Gao Y, Li J, Burgess R, Han J, Liang H, et al. A DNA binding winged helix domain in CAF-1 functions with PCNA to stabilize CAF-1 at replication forks. Nucleic Acids Res. 2016;44: 5083-94. doi:10.1093/nar/gkw106

26. Boeri-Erba E, Ochsenbein F, Sitbon D, Timm J, Mücke N, Sauer PV, et al. Insights into the molecular architecture and histone $\mathrm{H} 3-\mathrm{H} 4$ deposition mechanism of yeast Chromatin assembly factor 1. Elife. 2017;6. doi:10.7554/elife.23474

27. Mattiroli F, Gu Y, Yadav T, Balsbaugh JL, Harris MR, Findlay ES, et al. DNAmediated association of two histone-bound complexes of yeast Chromatin Assembly Factor-1 (CAF-1) drives tetrasome assembly in the wake of DNA replication. Elife. 2017;6. doi:10.7554/eLife.22799

28. Dovrat D, Dahan D, Sherman S, Tsirkas I, Elia N, Aharoni A. A Live-Cell Imaging Approach for Measuring DNA Replication Rates. Cell Rep. 2018;24: 252-258. doi:10.1016/j.celrep.2018.06.018

29. Dahan D, Tsirkas I, Dovrat D, Sparks MA, Singh SP, Galletto R, et al. Pifl is essential for efficient replisome progression through lagging strand G-quadruplex 
DNA secondary structures. Nucleic Acids Res. 2018; 1-11. doi:10.1093/nar/gky1065

30. Kaufman PD, Kobayashi R, Kessler N, Stillman B. The p150 and p60 subunits of chromatin assemblyfactor I: A molecular link between newly synthesized histories and DNA replication. Cell. 1995;81: 1105-1114. doi:10.1016/S00928674(05)80015-7

31. Mattiroli F, Gu Y, Balsbaugh JL, Ahn NG, Luger K. The Cac2 subunit is essential for productive histone binding and nucleosome assembly in CAF-1. Sci Rep. 2017;7: 1-11. doi:10.1038/srep46274

32. Game JC, Kaufman PD. Role of Saccharomyces cerevisiae chromatin assembly factor-I in repair of ultraviolet radiation damage in vivo. Genetics. 1999;151: 485497.

33. Sanchez Y, Bachant J, Wang H, Hu F, Liu D, Tetzlaff M, et al. Control of the DNA damage checkpoint by Chk1 and Rad53 protein kinases through distinct mechanisms. Science (80- ). 1999;286: 1166-1171. doi:10.1126/science.286.5442.1166

34. Weinert TA, Kiser GL, Hartwell LH. Mitotic checkpoint genes in budding yeast and the dependence of mitosis on DNA replication and repair. Genes Dev. 1994;8: 652665. doi:10.1101/gad.8.6.652

35. Amon A. The spindle checkpoint. Curr Opin Genet Dev. 1999;9: 69-75. doi:10.1016/S0959-437X(99)80010-0

36. Sharp JA, Franco AA, Osley MA, Kaufman PD. Chromatin assembly factor I and Hir proteins contribute to building functional kinetochores in S. cerevisiae. Genes Dev. 2002;16: 85-100. doi:10.1101/gad.925302

37. Su D, Hu Q, Li Q, Thompson JR, Cui G, Fazly A, et al. Structural basis for recognition of $\mathrm{H} 3 \mathrm{~K} 56$-acetylated histone $\mathrm{H} 3-\mathrm{H} 4$ by the chaperone Rtt106. Nature. 2012;483: 104-107. doi:10.1038/nature10861

38. Huang S, Zhou H, Katzmann D, Hochstrasser M, Atanasova E, Zhang Z. Rtt106p is a histone chaperone involved in heterochromatin-mediated silencing. Proc Natl Acad 
Sci. 2005;102: 13410-13415. doi:10.1073/pnas.0506176102

39. Zeman MK, Cimprich KA. Causes and consequences of replication stress. Nat Cell Biol. 2014;16: 2-9. doi:10.1038/ncb2897

40. Lisby M, Barlow JH, Burgess RC, Rothstein R. Choreography of the DNA damage response: Spatiotemporal relationships among checkpoint and repair proteins. Cell. 2004;118: 699-713. doi:10.1016/j.cell.2004.08.015

41. Huang TH, Fowler F, Chen CC, Shen ZJ, Sleckman B, Tyler JK. The Histone Chaperones ASF1 and CAF-1 Promote MMS22L-TONSL-Mediated Rad51 Loading onto ssDNA during Homologous Recombination in Human Cells. Mol Cell. 2018;69: 879-892.e5. doi:10.1016/j.molcel.2018.01.031

42. Hendel A, Krijger PHL, Diamant N, Goren Z, Langerak P, Kim J, et al. PCNA ubiquitination is important, but not essential for translesion DNA synthesis in mammalian cells. PLoS Genet. 2011;7. doi:10.1371/journal.pgen.1002262

43. Weill U, Yofe I, Sass E, Stynen B, Davidi D, Natarajan J, et al. Genome-wide SWAp-Tag yeast libraries for proteome exploration. Nat Methods. 2018;15: 617622. doi:10.1038/s41592-018-0044-9

44. Plate I, Hallwyl SCL, Shi I, Krejci L, Müller C, Albertsen L, et al. Interaction with RPA is necessary for Rad52 repair center formation and for its mediator activity. $\mathrm{J}$ Biol Chem. 2008;283: 29077-85. doi:10.1074/jbc.M804881200

45. Pardo B, Crabbé L, Pasero P. Signaling pathways of replication stress in yeast. FEMS Yeast Res. 2017;17. doi:10.1093/femsyr/fow101

46. Hedglin M, Benkovic SJ. Regulation of Rad6/Rad18 Activity During DNA Damage Tolerance. http://dx.doi.org/101146/annurev-biophys-060414-033841. 2015 [cited 27 Mar 2019]. doi:10.1146/ANNUREV-BIOPHYS-060414-033841

47. Ulrich HD, Walden H. Ubiquitin signalling in DNA replication and repair. Nat Rev Mol Cell Biol 2010 117. 2010;11: 479. doi:10.1038/nrm2921

48. Fridman Y, Palgi N, Dovrat D, Ben-Aroya S, Hieter P, Aharoni A. Subtle Alterations in PCNA-Partner Interactions Severely Impair DNA Replication and Repair. Haber JE, editor. PLoS Biol. 2010;8: e1000507. 
doi:10.1371/journal.pbio.1000507

49. Nyswaner KM, Checkley MA, Yi M, Stephens RM, Garfinkel DJ. Chromatinassociated genes protect the yeast genome from Ty1 insertional mutagenesis. Genetics. 2008;178: 197-214. doi:10.1534/genetics.107.082602

50. Avvakumov N, Nourani A, Côté J. Histone Chaperones: Modulators of Chromatin Marks. Mol Cell. 2011;41: 502-514. doi:10.1016/j.molcel.2011.02.013

51. Burgess RJ, Han J, Zhang Z. The Ddc1-Mec3-Rad17 sliding clamp regulates histone-histone chaperone interactions and dna replicationcoupled nucleosome assembly in budding yeast. J Biol Chem. 2014;289: 10518-10529. doi:10.1074/jbc.M114.552463

52. Hu F, Alcasabas AA, Elledge SJ. Asf1 links Rad53 to control of chromatin assembly. Genes Dev. 2001;15: 1061-6. doi:10.1101/gad.873201

53. Emili A, Schieltz DM, Yates JR, Hartwell LH. Dynamic interaction of DNA damage checkpoint protein Rad53 with chromatin assembly factor Asf1. Mol Cell. 2001;7: 13-20. Available: http://www.ncbi.nlm.nih.gov/pubmed/11172707

54. Mattiroli F, Gu Y, Yadav T, Balsbaugh JL, Harris MR, Findlay ES, et al. DNAmediated association of two histone-bound complexes of yeast chromatin assembly factor-1 (CAF-1) drives tetrasome assembly in the wake of DNA replication. Elife. 2017;6: 1-23. doi:10.7554/eLife.22799

55. Kim J-A, Haber JE. Chromatin assembly factors Asfl and CAF-1 have overlapping roles in deactivating the DNA damage checkpoint when DNA repair is complete. Proc Natl Acad Sci. 2009;106: 1151-1156. doi:10.1073/pnas.0812578106

56. Chen C-C, Carson JJ, Feser J, Tamburini B, Zabaronick S, Linger J, et al. Acetylated Lysine 56 on Histone H3 Drives Chromatin Assembly after Repair and Signals for the Completion of Repair. Cell. 2008;134: 231-243. doi:10.1016/j.cell.2008.06.035

57. Selth L, Svejstrup JQ. Vps75, a new yeast member of the NAP histone chaperone. J Biol Chem. 2007;282: 12358-12362. doi:10.1074/jbc.C700012200

58. Ito H, Fukuda Y, Murata K, Kimura A. Transformation of intact yeast cells treated with alkali cations. J Bacteriol. 1983;153: 163-8. Available: 
http://www.ncbi.nlm.nih.gov/pubmed/6336730

59. Soreanu I, Hendler A, Dahan D, Dovrat D, Aharoni A. Marker-free genetic manipulations in yeast using CRISPR/CAS9 system. Curr Genet. 2018;64: 11291139. doi:10.1007/s00294-018-0831-y

60. Fridman Y, Palgi N, Dovrat D, Ben-Aroya S, Hieter P, Aharoni A. Subtle alterations in PCNA-partner interactions severely impair DNA replication and repair. PLoS Biol. 2010;8. doi:10.1371/journal.pbio.1000507

61. Lea DE, Coulson CA. The distribution of the numbers of mutants in bacterial populations. J Genet. 1949;49: 264-285. doi:10.1007/BF02986080

62. Zheng Q. rSalvador: An R Package for the Fluctuation Experiment. G3\&amp;\#58; Genes|Genomes|Genetics. 2017;7: 3849-3856. doi:10.1534/g3.117.300120

63. Nishimura K, Fukagawa T, Takisawa H, Kakimoto T, Kanemaki M. An auxin-based degron system for the rapid depletion of proteins in nonplant cells. Nat Methods. 2009;6: 917-922. doi:10.1038/nmeth.1401

64. Morawska M, Ulrich HD. An expanded tool kit for the auxin-inducible degron system in budding yeast. Yeast. 2013;30: 341-351. doi:10.1002/yea.2967 